Esta revista forma parte del acervo de la Biblioteca Jurídica Virtual del Instituto de Investigaciones Jurídicas de la UNAM

\title{
CRÍTICA A LA PONDERACIÓN COMO TEST DE PROPORCIONALIDAD PARA DECIDIR SOBRE LA PUBLICIDAD DE LA INFORMACIÓN PERSONAL EN PODER DEL ESTADO EN MÉXICO
} REVIEW OF THE BALANCING RIGHTS TEST TO DETERMINE DISCLOSURE GOVERNMENT' INFORMATION IN MEXICO

\section{Juan Carlos UPEGUI MeJíA*}

\begin{abstract}
RESUMEN. Frente a la rigidez de las hipótesis legales para permitir la publicidad de la información personal en poder del Estado, el órgano de transparencia mexicano encontró una vía de excepción en la figura del interés público. La aparente ilegalidad de esta alternativa lo obligó a justificar su proceder y su competencia. Para ello, el órgano de transparencia se decantó por una lectura constitucional del problema mediante la figura de la ponderación. Después de contar esta historia, el autor analiza los usos de la ponderación, y argumenta que hay un aparente conflicto entre el derecho a la protección de datos personales y el derecho de acceso a la información pública, por lo que cuestiona la idoneidad del test de proporcionalidad para este fin.
\end{abstract}

PALABRAS CLAVE. Ponderación, test de proporcionalidad, colisión de derechos fundamentales, confidencialidad, información personal, interés público, acceso a la información pública.

ABSTRACT. Given the restrictive legal provisions for confidential information in Mexican' access to information legislation,

* Es doctorando en el Instituto de Investigaciones Jurídicas de la UNAM, maestro en derecho público y abogado por la Universidad Externado de Colombia,juan.upegui@ uexternado.edu.co.

Fecha de recepción: 21 de junio de 2017.

Fecha de dictamen: 17 de octubre de 2017 
Esta revista forma parte del acervo de la Biblioteca Jurídica Virtual del Instituto de Investigaciones Jurídicas de la UNAM

public interest principle of interpretation has become a way to determine document disclosure. The National Transparency and Access to Information Institute has used constitutional interpretation and proportionality balancing between access to information and privacy rights. The author argues that there is no rights conflict between access to information and privacy in many cases and that proportionality test is an inappropriate interpretation mechanism.

KEY WORDS. Proportionality and balancing rights, conflict of rights, privacy, access to information.

La metáfora de la ponderación no debería enmascarar el hecho de que la ponderación suele entrañar una discusión de razones saturada de teoría.

M. Кuмm

\section{Contexto normativo}

En México, desde 2002, la información personal en poder del Estado ha estado protegida por el mandato de confidencialidad. ${ }^{1}$ Esta regla general tiene cinco excepciones: cuatro de ellas presentes desde 2002: mandato legal u orden judicial, solicitud por autoridad competente, y figuración en registro público o fuente de acceso público; ${ }^{2}$ la quinta, el interés público, fue incluida por la Ley General de Transparencia en 2015, en los casos de seguridad nacional, salubridad general y protección de los derechos de terceros, y en el de "colisión de derechos". ${ }^{3}$

Según el marco regulatorio previo a la Ley General de Transparencia, el problema de la publicidad de la información personal en poder del

1 Artículo 18, Ley Federal de Transparencia y Acceso a la Información Pública Gubernamental (abrogada) (2002); artículo 116, Ley General de Transparencia y Acceso a la Información Pública (2015), y artículo 113, nueva Ley Federal de Transparencia y Acceso a la Información Pública (2016).

2 Artículos 18 y 22, Ley Federal de Transparencia (abrogada) (2002); artículo 120, Ley General de Transparencia (2015), y artículo 117, nueva Ley Federal de Transparencia (2016).

3 Artículos 120, fracción IV, y párrafo final, y 149, respectivamente. 
Esta revista forma parte del acervo de la Biblioteca Jurídica Virtual del Instituto de Investigaciones Jurídicas de la UNAM

Estado estaba prácticamente resuelto: la ley establecía con claridad su operación, y para ello bastaba seguir un sencillo procedimiento de subsunción. Una pregunta por los "métodos" para resolver este problema sería innecesaria, pues semejante problema no existía. Sin embargo, algunas decisiones del órgano federal de transparencia, entonces IFAI (ahora INAI), adelantaron la ruina de este ideal regulatorio, el cual terminaría por hacer aguas con la inclusión de la prueba de interés público y de la colisión de derechos en la Ley General de Transparencia. Esta reforma legislativa, sin embargo, no puede entenderse con independencia del trasegar del órgano de transparencia, y en especial de sus (polémicas) decisiones de exceptuar la confidencialidad de la información personal por fuera de los estrictos términos de la ley. ${ }^{4}$

\section{EXCEPCIONES A LA CONFIDENCIALIDAD DE LA INFORMACIÓN PERSONAL MÁS ALLÁ DE LA LEY}

De forma excepcional, el órgano de transparencia reconoció la posibilidad de hacer pública información personal, por fuera de las causales de ley, con base en dos argumentos: cuando la misma permitiese realizar los objetivos de transparencia gubernamental y rendición de cuentas de la (abrogada) Ley Federal de Transparencia, y cuando revistiese un interés público. Estos argumentos se emplearon para morigerar los efectos (injustos, irrazonables, desproporcionados, inoportunos, disfuncionales) del sistema cerrado de regla excepción. Con ellos se introdujeron excepciones a la regla de confidencialidad de toda la información personal cuando las circunstancias del caso indicaban que la información personal debería ser revelada.

Al emplear estas opciones, el órgano de transparencia se enfrentaba con dos problemas: la fundamentación de su competencia para exceptuar la confidencialidad, y la fundamentación material de la publicidad de la información personal en ausencia de un mandato legal específico.

La principal estrategia empleada por el órgano de transparencia para resolver el primero de los problemas fue la introducción de una lectura constitucional del problema de la publicidad de la información personal

4 Una de estas polémicas decisiones del IFAI fue la contenida en la resolución R 6030/09, CP Ángel Trinidad Zaldívar, en la que resuelve levantar la confidencialidad de información personal relativa a condonación de créditos fiscales por razones de interés público. Una aproximación crítica a esta decisión la realiza Issa Luna Pla y Gabriela Ríos Granados (2010: 39-71). 
Esta revista forma parte del acervo de la Biblioteca Jurídica Virtual del Instituto de Investigaciones Jurídicas de la UNAM

en poder del Estado. ${ }^{5}$ Esta lectura se concreta en la idea (recurrente) de que este tipo de casos es mejor entendido como una "colisión de derechos fundamentales" y no como uno sujeto a la aplicación estricta de la ley y la legislación secundaria (reglamento, lineamientos, normas oficiales, etcétera). La información personal en poder del Estado es de forma simultánea objeto de protección del derecho (fundamental) de acceso a la información pública y del derecho (fundamental) a la protección de datos personales. Por tanto, en los casos de acceso a la información personal en poder del Estado se presentaría una típica colisión de derechos. Una situación regulada por mandatos (constitucionales) contradictorios, donde no existe un criterio normativo de preferencia, y que escapa al sistema cerrado de regla excepción, definido por las leyes y por la legislación secundaria.

Una lectura constitucional del problema en estas coordenadas es susceptible de manipulación. En términos teóricos, todos los casos de acceso a la información personal en poder del Estado podrían diagnosticarse, entenderse y enfrentarse como casos de colisión entre los dos derechos fundamentales enunciados. De hacer carrera, esta idea haría inútil el sistema cerrado de regla-excepción con el que se ha intentado regular el problema de la publicidad de la información personal en poder del Estado. Y, además, impracticable y sumamente dispendioso el trabajo administrativo de clasificación y desclasificación de la información personal en poder del Estado.

Debía existir entonces un criterio que permitiese distinguir entre los casos que deben entenderse como casos de colisión de derechos y los que no. Este criterio parece descansar en el carácter de la información personal, y en específico, en el posible interés público que pueda revestir su comunicación. A pesar de que inicialmente esto estaba apenas sugerido, ${ }^{6}$

5 Como puede apreciarse en los casos de las resoluciones R 6030/09 (Luna y Ríos, 2010: 39-71) y R 1655/10, CP Ángel Trinidad Zaldívar (sobre la publicidad de la fotografía en título y cédula profesional de un servidor público), ambos con fundamento en un argumento de interpretación originalista del artículo 6o. constitucional. Según el IFAI, el Constituyente habría previsto que "fuera de las excepciones a la confidencialidad expresamente previstas en las leyes, determinados datos personales son susceptibles de divulgación si: 1) cuentan con un valor público y 2) las autoridades competentes realizan una cuidadosa ponderación que justifique la divulgación de los datos personales".

6 Como en los casos de las resoluciones R 3751/09, CP María Marván Laborde (sobre el interés público del expediente clínico de personas fallecidas durante su privación de la libertad en el penal "El Altiplano"), R 6030/09 (sobre el interés público en la información relativa a la condonación de créditos fiscales), R 5084/10 CP Jacqueline Peschard Mariscal (sobre el interés público de la información sobre militares condenados "por delitos contra la salud, crimen organizado y/o uso de recursos de procedencia ilícita”, cuya sentencia 
Esta revista forma parte del acervo de la Biblioteca Jurídica Virtual del Instituto de Investigaciones Jurídicas de la UNAM

no tardaría en aparecer de forma explícita como criterio fundante de la excepción a la regla de confidencialidad. ${ }^{7}$ Si se puede determinar que existe un interés público en la información personal, estamos en presencia de una (verdadera) colisión de derechos fundamentales, y el órgano de transparencia sería, en principio, el encargado de su resolución.

Asimismo, el concepto de interés público será clave para resolver el problema de la fundamentación material de la publicidad de la información personal en ausencia de un mandato legal específico. El argumento del órgano de transparencia ha sido que si se logra demostrar que la revelación de la información personal reviste un interés público y si este interés público tiene la entidad de derrotar el (supuesto) interés privado que existe en la confidencialidad de la información personal, habría una razón (material) suficiente para aceptar una excepción a la regla general de confidencialidad de la información personal, incluso por fuera de la ley.

Ambos problemas de fundamentación, tanto el de la competencia como el de la decisión de publicidad, terminan alineados en la definición de una metodología para resolver el problema de la publicidad de la información personal en poder del Estado. Visto así, en los casos diagnosticados como colisiones de derechos fundamentales, la pregunta por el problema de la publicidad de la información personal en poder del Estado es pertinente, y la cuestión de la metodología resulta central.

\section{Cuatro métodos}

El órgano de transparencia ha empleado al menos cuatro métodos para resolver el problema de la publicidad de la información personal en poder del Estado: subsunción, ponderación de daños, prueba de interés público y proporcionalidad.

estuviera en firme), R 1655/10 (véase la nota 5), RDA 178/13, CP María Elena Perez-Jaén Zermeño (sobre el interés público de la información contenida en un título profesional de un particular, en especial el voto disidente de Ángel Trinidad Zaldívar), RDA 4638/12, CP Ángel Trinidad Zaldívar (sobre el interés público de la información personal relativa a la evaluación de los docentes de primaria), entre otras.

7 Como se aprecia en la resolución RDA 2165/15, CP Areli Cano Guadiana (sobre la publicidad de la información personal relativa a condenas por el delito de lavado de activos), "[E]l interés público que tenga cierta información, será el concepto legitimador de las intromisiones en la intimidad o la vida privada, en donde el derecho a la intimidad o a la vida privada debe ceder a favor del derecho a comunicar y recibir información, [según] las circunstancias de cada caso concreto". 
Esta revista forma parte del acervo de la Biblioteca Jurídica Virtual del Instituto de Investigaciones Jurídicas de la UNAM

\section{Subsunción}

Este método, el más común y recurrido, parte de dos premisas: que las fronteras entre los derechos de acceso a la información pública y de protección de datos personales están bien delimitadas por el régimen jurídico aplicable, y que no existe, o no es posible, la "colisión" entre dichos dere40 chos. El método puede reducirse a la verificación de dos eventos: la presencia de información personal en la información solicitada, y la no existencia de una excepción (legal) a la confidencialidad. Si esto ocurre, está ordenada la clasificación de la información personal por confidencialidad.

- En los primeros casos sobre el acceso al expediente médico del presidente de la República es muy clara la aplicación de este método. ${ }^{8}$ También es muy clara en los casos de información personal de servidores públicos cuya confidencialidad no fue exceptuada por la ley. ${ }^{9} \mathrm{O}$ frente a la información personal cuya confidencialidad está explícitamente exceptuada en la ley, como en el caso de los beneficiarios de programas sociales ${ }^{10}$ La aplicación de este método es sencilla y se ajusta a la premisa según la cual prácticamente toda la información personal en poder del Estado se encuentra en la esfera privada y su publicidad está prohibida. Asimismo, es afín a los mecanismos de armonización concreta entre los derechos al acceso a la información pública y la protección de datos personales: la versión pública, la anonimización ${ }^{11}$ y la disociación de la información. ${ }^{12}$

\section{Ponderación de daños}

De uso esporádico, este método parte de la premisa de una colisión entre derechos, y consiste en ofrecer un criterio de preferencia depen-

8 R 1326/04, CP María Marván Laborde y R 425/09, CP Jacqueline Peschard Mariscal (confidencialidad de la información relacionada con la salud del presidente de la República).

9 R 751/11, CP Sigrid Arzt Colunga (confidencialidad de los datos personales de los servidores públicos, como estado civil, huella digital, nombres de los hijos, fecha de nacimiento, nacionalidad, referencias personales y número de matrícula militar, entre otros).

10 RDA 6302/15, CP Areli Cano Guadiana (publicidad de los nombres y demás datos personales de los adultos contenidos en el padrón de beneficiarios del seguro popular), y RDA 67/16, CP María Patricia Kurczyn Villalobos (publicidad de los nombres contenidos en el padrón de beneficiarios de Prospera).

11 RDA 621/12, CP Sigrid Arzt Colunga (anonimización de la información sobre auxilios entregados a las víctimas del incendio de la guardería ABC).

12 RDA 5599/15, CP Ximena Puente de la Mora (anonimización y disociación de la información sobre los listados de calificaciones de los estudiantes del ITC). 
Esta revista forma parte del acervo de la Biblioteca Jurídica Virtual del Instituto de Investigaciones Jurídicas de la UNAM

diendo de la relación de proporcionalidad que exista entre las magnitudes de daño que implicaría privilegiar uno u otro derecho. La estimación de los daños, sin embargo, no se adelanta de acuerdo con un sistema de medidas (valoración pecuniaria, por ejemplo), sino a partir de valoraciones sobre la importancia de los intereses en juego, por lo que termina asimilándose a la prueba de interés público.

Hay al menos tres alusiones aisladas a esta metodología. En un caso de acceso a la información del pasado se consideró que "la prueba del daño obliga a valorar el daño de la divulgación", que sólo puede apreciarse "frente a la ponderación del bien social de la desclasificación”. ${ }^{13}$ En el caso de la cancelación de créditos por parte del SAT, en donde consideró que "el daño" a la "privacidad fiscal no sería mayor al interés público por transparentar la manera en que el [SAT] determina cancelar créditos fiscales". ${ }^{14} \mathrm{Y}$ en el caso de la información de beneficiarios y montos de las ayudas a las víctimas de la guardería $\mathrm{ABC}$, en donde fungió como argumento concluyente para anonimizar la información, pues de lo contrario "podría producirse un daño mayor a las personas involucradas con la publicación de sus nombres, que al interés público por conocer[los]”. ${ }^{15}$

\section{Prueba de interés público}

Esta metodología parte de dos premisas: aceptar que el conocimiento de cierta información personal puede revestir un interés público, y que una vez que esto ha sido verificado, el caso debe ser entendido como una colisión entre derechos. La prueba consiste en aplicar la ley de ponderación a los intereses enfrentados: el interés del particular en mantener la confidencialidad de su información personal y el interés público en su revelación. Buenos ejemplos de la aplicación de esta metodología son el caso de la información contenida en la cédula y el título profesional, en el cual el IFAI precisó, primero, "determinar si es de interés público el conocimiento" del dato personal, y, segundo, "realizar una ponderación entre el derecho del titular [del dato personal] para que se guarde la confidencialidad... y el derecho de

13 R 412/06 y R 413/06 acumulados, CP Alonso Lujambio Irazábal, en especial el voto particular de Juan Pablo Guerrero Amparán (publicidad y confidencialidad de cierta información sobre Octavio Paz y Elena Garro, contenida en la Galería 1 del Archivo General de la Nación).

14 R 6030/09 (Luna y Ríos, 2010: 39-71).

15 RDA 621/12 (véase la nota 11). 
Esta revista forma parte del acervo de la Biblioteca Jurídica Virtual del Instituto de Investigaciones Jurídicas de la UNAM

acceso a la información de los ciudadanos”, ${ }^{16}$ y además, uno de los casos relacionados con información sobre cancelación de créditos del SAT, en donde consideró que conocer la información personal "abonaría a la valoración del desempeño del sujeto obligado... [lo cual] tiene un interés público mayor al de la protección de los datos fiscales”. ${ }^{17}$

Hay tres antecedentes de la prueba de interés público en la práctica del entonces IFAI. El primero es el caso sobre la confidencialidad de las fotografías de servidores públicos, en donde aparece, paradójicamente, como un argumento de refuerzo para mantener la confidencialidad de dichas fotos, al no verificar "un interés público mayor en conocer esta información al interés en proteger estos datos personales". ${ }^{18}$ El segundo está contenido en los casos sobre la información del pasado, en donde se utiliza la figura del "test de interés social" para exceptuar la confidencialidad de la información personal. Este "test" incluía tres variables: el paso del tiempo, la posibilidad de gradar la información personal y el argumento teleológico orientado a la verdad histórica. El test se concreta con una aplicación de la ley de ponderación, según la cual "conocer las circunstancias que rodearon los movimientos sociales y políticos del pasado... resulta de mayor importancia" frente "a la posible afectación [de la] intimidad". ${ }^{19}$ Finalmente, está el primer caso de información personal sobre cancelación de créditos fiscales, en donde la prueba de interés público ya estaba perfilada, a pesar de que no fue aplicada al caso concreto. ${ }^{20}$

La construcción discursiva de la prueba de interés público es un ejemplo fascinante de cómo el órgano de transparencia intenta morigerar los efectos de la rigidez de la regla general de la confidencialidad de toda la información personal en poder del Estado. Para su fundamentación ha empleado tres tipos de argumentos: el teleológico, según el cual la información personal debe darse a la publicidad si contribuye a la realización de la transparencia y la rendición de cuentas; el originalista, de acuerdo

16 R 1655/10 (véase la nota 5).

17 RDA $1118 / 15$, CP Rosendoevgueni Monterrey Chepov (publicidad del número de créditos fiscales firmes [no pagados y no garantizados] y los montos por créditos cancelados o condonados a favor de ciertas personas físicas y morales en un periodo de tiempo).

18 R 934/05, CP Juan Pablo Guerrero Amparán (publicidad de la fecha de nacimiento y cierta información del currículo de los servidores públicos, y confidencialidad de la fotografía).

19 R 412/06 y R 413/06 acumulados (véase la nota 13), y R 522/06, CP Alonso Lujambio Irazábal (publicidad de información relativa a cuatro personas que fueron víctimas del delito de desaparición forzada durante la "guerra sucia”).

${ }^{20}$ R 6030/09 (Luna y Ríos, 2010: 39-71). 
Esta revista forma parte del acervo de la Biblioteca Jurídica Virtual del Instituto de Investigaciones Jurídicas de la UNAM

con el cual el propio constituyente, al introducir la reforma al artículo 6o. constitucional, habría previsto que "fuera de las excepciones a la confidencialidad expresamente previstas en las leyes, determinados datos personales son susceptibles de divulgación si: 1) cuentan con un valor público y 2) las autoridades competentes realizan una cuidadosa ponderación que justifique [su] divulgación”, ${ }^{21}$ y, finalmente, el argumento pragmático, acerca de una cierta necesidad de resolver la "colisión" entre los derechos al acceso a la información pública y la protección de datos, cuando se presentan "casos frontera" en donde es "difícil discernir... cuándo termina uno y comienza el otro... o en qué supuestos debe ceder uno frente al otro". 22

\section{El test de proporcionalidad}

Este método es una adaptación de la teoría de los principios de Robert Alexy para resolver el problema de la publicidad de la información personal en poder del Estado. En la práctica del IFAI-INAI consta de dos pasos: la identificación o el diagnóstico del caso como una "colisión" entre derechos fundamentales y el despliegue de sus tres subprincipios (idoneidad, necesidad y proporcionalidad en sentido estricto) que sirven para enmarcar y dirigir la argumentación. Entre los primeros casos en que se empleó esta metodología pueden citarse el de la publicidad de condenas de militares y el de la confidencialidad de las víctimas del incendio de la guardería ABC. En ambos casos, la inspiración del IFAI fue una tesis del Poder Judicial de la Federación. ${ }^{23}$

Para 2015, aumenta la frecuencia del uso del test de proporcionalidad, como puede advertirse de la suma de los casos sobre evaluación docente, ${ }^{24}$ condenados por delitos de lavado de activos, de alto impacto y

${ }^{21}$ R 6030/09 (Luna y Ríos, 2010: 39-71) y R 1655/10 (véase la nota 5).

22 RDA 4638/12 (véase la nota 6).

23 R 5084/10 (véase la nota 6) considerando quinto, a), y RDA 621/12 (véase la nota 11), considerando octavo. "Tesis I.4o.A.70 K emitida por el Cuarto Tribunal Colegiado en Materia Administrativa del Primer Circuito. Novena Época, localizable en el Semanario Judicial de la Federación y su Gaceta, XXIV, agosto de 2006, página 2346. Suspensión en el amparo. Conforme a la teoría de ponderación de principios debe negarse si el interés social constitucionalmente tutelado es preferente al del particular" (la forma de citar este criterio no es uniforme en las dos resoluciones, utilicé la cita de la primera resolución).

24 RDA 1312/15, CP Oscar Mauricio Guerra Ford (publicidad de los resultados de la evaluación a los docentes de primaria). 
Esta revista forma parte del acervo de la Biblioteca Jurídica Virtual del Instituto de Investigaciones Jurídicas de la UNAM

de terrorismo, ${ }^{25}$ servidores públicos condenados, ${ }^{26}$ confidencialidad de la identidad de niños y niñas en el padrón del seguro popular ${ }^{27}$ y sanciones a médicos. ${ }^{28}$

La adaptación de la teoría de los principios camina hacia la estandarización en las prácticas del INAI en un ejercicio de sincretismo metodológico. El método resultante es más o menos el siguiente: se afirma el interés público de cierta información personal, se diagnostica el caso como una colisión de derechos fundamentales, se plantea su resolución por medio de la ponderación (hasta aquí parecería que es la misma prueba de interés público), y para adelantar el ejercicio de "ponderación" se utilizan los subprincipios del test de proporcionalidad.

El uso de la terminología de la teoría de los principios tiene dos funciones en esta fórmula ecléctica: organizar la argumentación y legitimar la decisión. Una lectura detenida de lo que hace realmente el órgano de transparencia cuando despliega el test lo ilustra. Tomemos los casos de la evaluación docente ${ }^{29}$ y de los médicos sancionados por la Conamed ${ }^{30}$ como ejemplos paradigmáticos.

En el "análisis" de la idoneidad, el INAI ubica por lo general un argumento teleológico (si la publicidad de la información cumple el fin de realizar un interés público). En el caso de los docentes: "al restringirse la protección de los datos personales de... los docentes... se tendría el conocimiento objetivo... de la capacidad de los profesores evaluados". En el caso de los médicos, "existe un fin constitucionalmente válido para dar a conocer el nombre y cédula del médico que obtuvo un laudo condenatorio... que es [sic] el derecho fundamental a la salud". En el "análisis" de necesidad ubica un argumento empírico de la relación medio fin (si existen o no otros medios menos lesivos para realizar el interés público). En el caso

25 RDA 2165/15 (véase la nota 7); RDA 2445/15, CP Areli Cano Guadiana (publicidad de la información relativa a personas extraditadas condenados por delitos de "impacto trascendente en la sociedad", a saber: "delincuencia organizada, contra la salud, portación de armas de fuego de uso exclusivo del Ejército y Fuerzas Armadas y secuestro"); RDA 6206/15, CP María Patricia Kurzcyn Villalobos (publicidad de la información relativa a condenados por el delito de financiación del terrorismo).

26 RDA 5112/15 CP Areli Cano Guadiana (publicidad sobre condenas en firme de servidores públicos).

27 RDA 6302/15 (véase la nota 10) (confidencialidad de los nombres y demás datos personales de niños y niñas contenidos en el padrón de beneficiarios del seguro popular).

28 RDA 6531/15, CP Joel Salas Suárez (publicidad de nombres y números de cédula profesional de médicos, contenidos en laudos condenatorios expedidos por la Conamed).

29 RDA 1312/15 (véase la nota 24), considerando cuarto.

30 RDA 6531/15 (véase la nota 28), considerando quinto. 
Esta revista forma parte del acervo de la Biblioteca Jurídica Virtual del Instituto de Investigaciones Jurídicas de la UNAM

de los docentes "no existe otro medio de acceso menos lesivo al derecho a la protección de los datos personales... que el de obtener dicha información a través del procedimiento de acceso a la información”. En el caso de los médicos: "No existe un medio menos oneroso... [que facilitar] que las personas estén en aptitud de conocer qué médicos han sido sujetos de un laudo condenatorio por una mala praxis". Y finalmente, en el "análisis" de proporcionalidad en sentido estricto ubica un argumento de prevalencia (si el interés público satisfecho es más importante que la pérdida de privacidad). En el caso de los docentes, "el beneficio de dar a conocer los resultados de los docentes... es mayor que la lesión que se pueda causar a la esfera de protección de datos personales... porque la educación otorgada por el Estado tiene consecuencias... en el desarrollo económico, político y social del país". Y en el caso de los médicos, "El sacrificio de la protección al nombre y cédula de los médicos referidos... se justifica debido a que se satisface el interés ciudadano en conocer al personal médico que haya incurrido en una mala práctica profesional causando afectaciones al derecho fundamental a la salud de sus pacientes". ${ }^{31}$

\section{EL CONCEPTO ESQUiVO DE PONDERACIÓN}

La idea de ponderación es recurrente en la argumentación del órgano de transparencia. Esta idea aparece alineada con la del método para resolver los problemas de publicidad de la información personal en los casos de colisión entre derechos fundamentales; sin embargo, el órgano de transparencia utiliza la expresión "ponderación” en diversos sentidos. El carácter ambiguo de la expresión "ponderación” y su uso diverso tiene efectos relevantes para la buena comprensión de la competencia, de la metodología para resolver los casos y de la función del concepto para definir el contenido de los derechos en colisión.

El órgano de transparencia ha utilizado la expresión ponderación como: i) sinónimo de la armonización concreta, como sucede en los casos del acceso al expediente médico de presos fallecidos ${ }^{32}$ y de la evalua-

31 Esta forma de organizar los argumentos se repite en las otras decisiones en las que el INAI dice adelantar un "ejercicio de ponderación". Por obvias razones de espacio, no las hacemos explícitas.

32 R 3751/09 (véase la nota 6), considerando, quinto. "[Un] ejercicio de ponderación implica atender a los bienes y valores jurídicos que en el caso concreto están relacionados y atender a sus propiedades relevantes para arribar a una determinación correcta... [que asegure] que coexistan y se ejerzan plenamente todos los derechos involucrados, mediante 
ción docente; ${ }^{33}$ ii) como una metodología para definir el contenido de los derechos fundamentales, como sucede en el caso de la guardería ABC; 34 iii) como expresiones ligeramente diferentes del criterio para definir la prelación normativa, también conocido como "ley de ponderación", como sucede en el caso de la publicidad de la información personal contenida en el título y la cédula profesional, ${ }^{35}$ en el de la evaluación docente ${ }^{36}$ o en el de los condenados por el delito de lavado de activos, ${ }^{37}$ y iv) como sinónimo del test de proporcionalidad, como sucede en los casos de la revelación de información de personas condenadas por delitos de lavado de activos, de alto impacto y de terrorismo, ${ }^{38}$ de sanciones a médicos ${ }^{39}$ y de confidencialidad

interpretaciones extensivas que permitan su manifestación con toda la fuerza expansiva que corresponde a los derechos humanos".

33 RDA 4638/12 (véase la nota 6), considerando séptimo. "[C]uando un derecho humano entra en colisión con otro, debe buscarse el justo medio a través de un ejercicio de ponderación, con miras a obtener el mayor beneficio de uno y el menor perjuicio posible para el otro, sin que en forma alguna pueda llevar al extremo de anular uno frente al otro".

34 RDA 621/12 (véase la nota 11), considerando quinto. "[El derecho a la protección de datos personales refiere] a bienes jurídicos muy genéricos o conceptos esencialmente controvertidos, en virtud de que tienen alcances no unívocos, poco asibles o de sentido equívoco, de ahí que a fin de establecer su contenido se debe realizar un ejercicio de ponderación jurídica en el que se atienda a la necesidad, idoneidad y proporcionalidad de la restricción de que se trata”.

35 R 1655/10 (véase la nota 5), considerando quinto, numeral 3. La cita al Dictamen de las Comisiones Unidas de Puntos Constitucionales y de la Función Pública por el que se reforma el artículo 6o. de la Constitución, de la Cámara de Diputados. "En otros casos, la ley deberá prever la posibilidad de que, algunos datos personales, puedan ser divulgados cuando un órgano jurisdiccional o administrativo determine que existen razones particulares que justifiquen su divulgación, previa garantía de audiencia del implicado. De cualquier forma, las autoridades deberán realizar una cuidadosa ponderación que justifique el hecho de que una información que pertenece al ámbito privado, puede ser divulgada por así convenir al interés público".

36 RDA 1312/15 (véase la nota 24), considerando cuarto. "La ponderación es la forma en que se aplican los principios jurídicos, es decir, las normas que tienen estructura de mandatos de optimización... La ponderación conlleva a realizar un ejercicio en el cual quede demostrado que... si bien existe un conflicto entre dos principios, la determinación que se adopta al darle preferencia a un derecho sobre el otro nos permite tener una ganancia frente a la restricción del otro... en principio", "Cuanto mayor es el grado de la no satisfacción o de afectación de uno de los principios, tanto mayor debe ser la importancia de la satisfacción del otro".

37 RDA 6206/15 (véase la nota 25), considerando cuarto. "[Resulta] imperioso realizar una ponderación [entendida como un] procedimiento de decisión, en donde la ley de ponderación exige que, a mayor intensidad de la intervención, las razones para la afectación deben tener un peso mayor".

38 RDA 2165/15 (véase la nota 7); RDA 2445/15 (véase la nota 25); RDA 6206/15 (véase la nota 25).

39 RDA 6531/15 (véase la nota 28). 
Esta revista forma parte del acervo de la Biblioteca Jurídica Virtual del Instituto de Investigaciones Jurídicas de la UNAM

de la identidad de niños y niñas en el padrón del seguro popular. ${ }^{40}$ El uso diverso de la expresión "ponderación" tiene, sin embargo, una constante: es empleada de forma concurrente con la del diagnóstico del problema de la publicidad de la información personal en poder del Estado como una colisión entre derechos fundamentales.

No existe definición estipulativa de "ponderación" en el ordenamiento jurídico mexicano. Sus usos y sus definiciones son objeto de la doctrina ${ }^{41}$ y de la jurisprudencia. El concepto de "ponderación" ha sido utilizado por Robert Alexy como parte del elenco conceptual de su teoría de los principios en al menos dos sentidos: el primero y más elemental es el que lo asocia a la llamada "ley de ponderación", según la cual "la medida permitida de no satisfacción o de afectación de uno de los principios depende del grado de importancia de la satisfacción del otro" (Alexy, 1997). El segundo, y más complejo, es el que identifica la ponderación con "el método" para resolver las colisiones entre derechos fundamentales, y que incluye dos herramientas: una escala tríadica (leve, media, alta) para la valoración de la afectación y la satisfacción de los principios en conflicto, y una sofisticada "fórmula del peso" (Alexy, 2003) que permita expresar "el peso de un principio en las circunstancias de un caso concreto" (Alexy, 2003), y que incluye varias reglas para definir la prelación entre los derechos fundamentales en colisión en un caso concreto, ${ }^{42}$ y de paso definir el contenido normativo de los mismos a partir de la identificación de "normas adscriptas" (Alexy, 1997; Bernal, 2003; Mendoza, 2007).

40 RDA 6302/15 (véase las notas 10 y 27).

41 En el ámbito europeo, el autor más influyente ha sido Robert Alexy, con su Teoría de los derechos fundamentales, trad. Ernesto Garzón Valdés, Madrid, Centro de Estudios Constitucionales, 1997. En el ámbito latinoamericano ha sido influyente la obra de Carlos Bernal, El principio de proporcionalidad y los derechos fundamentales, Madrid, Centro de Estudios Constitucionales, 2003, y los trabajos editados por Miguel Carbonell, El principio de proporcionalidad y la interpretación de la Constitución, Quito, Ministerio de Justicia y Derechos Humanos, 2008, y Neoconstitucionalismo(s), Madrid, Trotta, 2005. En el ámbito anglosajón, la crítica introducida por Aleinikoff sigue teniendo algo de vigencia; Aleinikoff, T. Alexander, "Constitutional Law in the Age of Balancing", The Yale Law Journal, vol. 96, núm. 5, abril de 1987), apesar de las voces que claman por un uso más intensivo y también más técnico de la figura, como Jackson, Vicki C., "Constitutional Law in an Age of Proportionality”, The Yale Law Journal, vol. 124, núm. 8, junio de 2015). Emplearé la conceptualización de Robert Alexy, por ser el primero en formular una teoría consistente sobre el problema de la ponderación y por su carácter de teoría canónica.

42 Las variables de la fórmula del peso provienen de tres tipos de argumentos. El primero relativo al valor asignado a la afectación y satisfacción de los principios enfrentados, el segundo relativo al llamado peso abstracto de cada uno de los principios enfrentados, y el tercero relativo a la fiabilidad de las premisas empíricas sobre la satisfacción y la afectación de los principios enfrentados. 
Esta revista forma parte del acervo de la Biblioteca Jurídica Virtual del Instituto de Investigaciones Jurídicas de la UNAM

Sin embargo, el concepto de "ponderación" no es la provincia exclusiva de la teoría de los principios de Alexy. El concepto se vale de la metáfora de la balanza, tan antigua como la idea misma de justicia, para aludir a una suerte de procedimiento o de criterio para decidir la prelación de una causa, un interés o un derecho en una situación concreta (Aleinikoff, 1987; Nils Jansen, 2011). Es un concepto propio del razonamiento práctico general, utilizado por distintas culturas en distintos escenarios y distintos momentos para resolver problemas prácticos.

Esta doble connotación del término "ponderación", como término especializado de la teoría de los principios y como término de uso común en - el escenario del razonamiento práctico general, permitiría explicar la mul- tiplicidad de sentidos en que es empleado por el órgano de transparencia. A la práctica de una recepción no necesariamente "pura" del concepto, tal y como es entendido en el contexto y con los fundamentos filosóficos de la teoría de los principios de Alexy (1997), se suma el uso común de la expresión propia del razonamiento práctico general. El pretendido carácter técnico de la expresión queda doblegado por su poderosa carga emotiva. El uso de la expresión ponderación tiene en las prácticas del órgano de transparencia esta doble prosapia.

\section{El elemento vernáculo}

El órgano de transparencia empieza a utilizar el concepto de ponderación bajo la forma del criterio normativo "del interés mayor" en el contexto específico de las excepciones a la confidencialidad de la información personal: bajo la fórmula de advertir en la revelación de la información personal un interés público mayor al interés privado en resguardarla. ${ }^{43}$

Una forma típica del argumento por ponderación propio del razonamiento práctico general, que es a la vez una expresión (rudimentaria) de la llamada "ley de ponderación". Esta forma de argumentar es temprana, y ya está presente en las resoluciones de 2005 del órgano de transparencia. ${ }^{44}$

$43 \mathrm{Al}$ pronunciarse sobre el acceso a cierta información personal, contenida en la galería 1 del Archivo General de la Nación, el IFAI consideró "de mayor importancia conocer quiénes fueron las personas que se encontraron involucradas en los movimientos sociales y políticos del pasado y la actuación de las autoridades respecto de ellas, que la posible afectación a su intimidad”. R 522/06 (véase la nota 19), considerando séptimo.

44 R 934/05 (véase la nota 18), considerando séptimo. "[E]s claro que la fotografía de un servidor público no refleja su desempeño ni acredita su idoneidad en el cargo, por lo que no puede decirse que exista un interés público mayor en conocer esta información al interés en proteger estos datos personales". 
Esta revista forma parte del acervo de la Biblioteca Jurídica Virtual del Instituto de Investigaciones Jurídicas de la UNAM

Este criterio se potenciará con la inclusión a las prácticas del órgano de transparencia de la llamada prueba de interés público hacia 2009. En su concepción original, la prueba de interés público tiene dos elementos: la constatación de que "x" información personal reviste interés público, y la presentación de razones por las cuales satisfacer el interés público es más importante que satisfacer el interés privado. ${ }^{45}$ El segundo de los elementos de la prueba de interés público está construido sobre una muy clara adaptación de la ley de ponderación como criterio normativo de preferencia.

\section{El trasplante como punto de inflexión}

Mientras se aviva el debate sobre la plausibilidad de la prueba de interés público (López Ayllón, 2007), ${ }^{46}$ aterriza en las prácticas del IFAI la idea de ver estos problemas como colisión de derechos fundamentales, y la necesidad de resolverla mediante ejercicios de ponderación. Lo interesante es que esta idea llega por un predecible intermediario: el Poder Judicial de la Federación. Desde 2010, el entonces IFAI invoca una tesis aislada de un tribunal administrativo que decidió, en un caso de amparo, resolver un problema de "colisión de derechos fundamentales" conforme al "criterio de proporcionalidad, ponderando los elementos o subprincipios siguientes: a) idoneidad .. b) necesidad... c) y el mandato de proporcionalidad entre medios y fines". ${ }^{47}$ Con base en estos novedosos "elementos", el IFAI adelanta el ejercicio de "ponderación" de los derechos al acceso a la información pública y a la protección de datos personales, y resuelve exceptuar el mandato de confidencialidad de la información personal relacionada con condenas a personal militar que estuvieran en firme ${ }^{48} \mathrm{y}$

45 R 6030/09 (Luna y Ríos, 2010: 39-71), considerando séptimo. "[A]ún en el supuesto de que las personas físicas y morales contaran con la protección del secreto fiscal - hecho que quedó desacreditado en el anterior considerando- el daño a su privacidad fiscal no sería mayor al interés público por transparentar la manera en que el [SAT] determina cancelar créditos fiscales".

46 López-Ayllón, Sergio y Posadas, Alejandro, "Las pruebas de daño e interés público en materia de acceso a la información. Una perspectiva comparada", Derecho Comparado de la Información, núm. 9, enero-junio de 2007, pp. 21-65, 54, "[N]i del lenguaje de la [Ley Federal de Transparencia] ni de su interpretación puede desprenderse que la LAI estableció la posibilidad de realizar una prueba de interés público en caso de la información confidencial".

47 Véase en especial la nota 23 con la referencia de la tesis jurisprudencial seguida por el IFAI en los casos R 5084/10 (véase la nota 6) y RDA 62 1/12 (véase la nota 11).

48 R 5084/10 (véase la nota 6). 
Esta revista forma parte del acervo de la Biblioteca Jurídica Virtual del Instituto de Investigaciones Jurídicas de la UNAM

el mandato de publicar los nombres de las personas que habían recibido recursos oficiales, provenientes del fondo para la indemnización de los afectados con el incendio de la guardería ABC. ${ }^{49}$

\section{El uso recurrente y estandarizado del test de proporcionalidad}

Desde entonces, la incorporación del test de proporcionalidad y sus subprincipios al elenco argumentativo del órgano de transparencia es incuestionable, y su uso es relativamente frecuente, como puede desprenderse de los casos reseñados de 2015. En estos casos hay indicios de la influencia de la teoría de los principios de Alexy, como se puede advertir en la cita que hace el órgano de transparencia al texto de Alejandro Nava, así como de la filosofía del derecho de Robert Alexy, en los casos sobre condenados por el delito de lavado de activos y por delitos de "impacto trascendente en la sociedad", y sobre médicos sancionados por la Conamed, ${ }^{50}$ y al texto del propio Robert Alexy, La teoría de los derechos fundamentales, en uno de los casos de la evaluación docente..$^{51}$ Estas citas parecen cumplir una función legitimadora del uso de esta figura, pues el órgano de transparencia no dialoga con estos autores, no los emplea para fundamentar el uso específico de estas categorías, ni para cuestionarlos, ni para precisarlos. El criterio fundamental sigue siendo la tesis aislada del Poder Judicial, con todo lo atinada o precaria que pueda ser.

49 RDA 621/12 (véase la nota 11).

50 Con cita expresa al texto de Alejando Nava Tovar, el INAI ha considerado que "[E]n un sistema jurídico racional, el contenido de ciertos derechos fundamentales no puede ser absoluto, y la colisión entre derechos fundamentales no puede resolverse apelando a reglas de prioridad entre normas, sino mediante una ponderación que determine el derecho que ha de prevalecer en el caso concreto". La referencia completa es la siguiente: "Nava Tovar, Alejandro, La institucionalización de la razón. La filosofía del derecho de Robert Alexy, México, Universidad Autónoma Metropolitana, 2015, p. 174”. Así, en las resoluciones RDA 2165/15 (véase la nota 7), considerando cuarto; RDA 2445/15 (véase la nota 25), considerando cuarto, y RDA 6531/15 (véase la nota 28), considerando quinto.

51 Con una notable cita a la ley de ponderación: "[E]s inminente advertir que la determinación que se toma al ponderar dos derechos de esta naturaleza sin duda afecta en medida [sic] a uno de ellos", es decir, en principio, "Cuanto mayor es el grado de la no satisfacción o de afectación de uno de los principios, tanto mayor debe ser la importancia de la satisfacción del otro". La referencia completa es la siguiente: Alexy, Robert, Teoría de los derechos fundamentales, trad. de Ernesto Garzón Valdés, Madrid, Centro de Estudios Políticos y Constitucionales, 1997, p. 161. Así, en la RDA 1312/15 (véase la nota 24), considerando cuarto. 
Esta revista forma parte del acervo de la Biblioteca Jurídica Virtual del Instituto de Investigaciones Jurídicas de la UNAM

No obstante el uso de la terminología del test de proporcionalidad y su rimbombante despliegue persiguiendo la "ponderación", una lectura detallada de cómo se utilizan estos términos, como ya lo vimos, revela que su función principal es más retórica que de fondo, más de legitimación que de fundamentación. El criterio que resuelve los casos sigue siendo la existencia o no de un interés público en la información personal, y el examen de la importancia de ese interés público frente al interés privado de mantener en secreto la información personal. Esto es, la nuda prueba de interés público.

\section{LA POCA UTILIDAD DEL TEST DE PROPORCIONALIDAD}

Dejando de lado sus funciones legitimadoras, el test de proporcionalidad es de poca utilidad para resolver el problema de la publicidad de la información personal en poder del Estado. El uso de los subprincipios de idoneidad y necesidad lleva al órgano de transparencia a forzar los argumentos o a emplear argumentos inocuos. Esto es así por dos razones: porque el test de proporcionalidad, entendido como criterio para valorar la constitucionalidad de las restricciones a los derechos fundamentales (Bernal, 2003), no fue diseñado para resolver colisiones de derechos fundamentales en casos concretos, y porque los subprincipios de idoneidad y necesidad son irrelevantes en este particular escenario, donde existe una clara polaridad entre los intereses enfrentados: publicidad-privacidad. Estas circunstancias no han sido valoradas por el órgano de transparencia que, en su lugar, ha hecho una aplicación acrítica de la herramienta, inducido por la ya mencionada tesis aislada del Poder Judicial de la Federación. Al resolver las supuestas colisiones entre los derechos fundamentales al acceso a la información pública y a la protección de datos personales, tanto el "análisis" de idoneidad como el de necesidad llevan al órgano de transparencia a la inclusión de argumentos inocuos y forzados.

Si aceptamos que el subprincipio de idoneidad supone un análisis de medio a fin, esto es, la estimación en el plano empírico de la relación de causalidad entre la medida que limita el derecho fundamental y la satisfacción del principio que la justifica (Alexy, 1997; Bernal, 2003), en donde "la constatación de que un medio es idóneo para alcanzar un fin requiere un pronóstico y únicamente un pronóstico; en ella no hay nada que valorar" (Schlink, 2014), la única conclusión posible es que en todo caso de colisión entre los derechos de acceso a la información pública y de protección de datos personales, la revelación de información personal (por mínima o 
Esta revista forma parte del acervo de la Biblioteca Jurídica Virtual del Instituto de Investigaciones Jurídicas de la UNAM

insignificante que sea) satisface, por definición, el derecho al acceso a la información pública. Esta conclusión también es cierta si se invierten los términos: la reserva de la información personal en todos los casos satisface, por definición, el derecho a la protección de datos personales. Si esto es así en todos los casos posibles, el supuesto "análisis" de idoneidad es totalmente prescindible por la sencilla razón de que no hay análisis alguno que realizar.

Lo mismo sucede si aceptamos que el subprincipio de necesidad supone un análisis entre los medios disponibles, como la estimación en el plano empírico de la existencia de otras medidas para satisfacer el princi- pio que las justifica y que sean igualmente idóneas para realizarlo (Alexy, - 1997; Bernal, 2003). En el conflicto entre derechos fundamentales, "si solamente existen otros medios que van ciertamente a favor de uno, pero en perjuicio de otro, se ha agotado el potencial del examen de necesidad" (Schlink, 2014). En nuestro caso, no existe otra medida que sea igualmente idónea para satisfacer el derecho al acceso a la información pública que la publicación de la información. O en el otro sentido, no existe otra medida que sea igualmente idónea para satisfacer el derecho a la protección de datos que guardar la confidencialidad. Si la idoneidad de la medida en uno y otro caso siempre serán o la publicidad o la confidencialidad, no hay que adelantar un "análisis" de idoneidad para llegar a una conclusión que está dada de antemano.

La polaridad de las medidas (publicidad-confidencialidad) con las que se resuelve la supuesta colisión entre los derechos fundamentales al acceso a la información pública y a la protección de datos personales nos lleva a concluir que si en todos los casos la medida que ordena la publicidad de la información personal es idónea y necesaria para satisfacer el derecho de acceso a la información pública, o la que ordena la confidencialidad es idónea y necesaria para satisfacer el derecho a la protección de datos, los supuestos "análisis" de idoneidad y de necesidad son prescindibles por superfluos.

Esta situación es crítica por dos razones: porque el órgano de transparencia suele ocupar una parte importante de su tiempo, de sus recursos y de su energía argumentativa en estos ejercicios y, además, porque al ser la máxima autoridad en la materia sus decisiones procedimentales tienen un efecto multiplicador sobre los sujetos obligados que resuelven este tipo de casos. Pero sobre todo es crítica, porque el legislador, en la Ley General de Transparencia, hizo obligatoria la aplicación del principio de proporcionalidad, incluidos los subprincipios de idoneidad y ne- 
Esta revista forma parte del acervo de la Biblioteca Jurídica Virtual del Instituto de Investigaciones Jurídicas de la UNAM

cesidad cuando, al resolver recursos de revisión, "exista una colisión de derechos". 52

Queda a salvo, sin embargo, el subprincipio de proporcionalidad, o la proporcionalidad en estricto sentido. El órgano de transparencia está familiarizado con la aplicación de la ley de ponderación o de sus múltiples variantes. Esto ha sido así al menos desde 2005, mucho antes de que el IFAI empezara a utilizar los conceptos de la teoría de los principios. El mejor ejemplo de esta práctica es la elaboración de la prueba de interés público y su aplicación efectiva en un par de casos. ${ }^{53}$

Esta herencia, sin embargo, permanecerá intacta, a pesar de la incorporación de la terminología del test de proporcionalidad y del (aparente) desplazamiento de la prueba de interés público. Esto es evidente en los casos resueltos en 2015, en donde se empleó el test de proporcionalidad para justificar la publicidad excepcional de cierta información personal. En estos casos, el INAI no acude, por ejemplo, a la fórmula del peso, el conjunto organizado de criterios ofrecido por Alexy para defender la racionalidad de la ponderación y de la aplicación del subprincipio de proporcionalidad en sentido estricto. El INAI continúa aplicando la ley de ponderación o sus variantes, como lo hace desde el 2005, bajo la fórmula gramatical del "interés mayor" y el supuesto de que la difusión de la información personal en cuestión reviste "interés público", con un uso por lo general razonable. Esta práctica también parece haber impregnado la particular concepción del test de proporcionalidad que trae la Ley General de Transparencia, en donde es claro el influjo de la experiencia del órgano de transparencia, dada la centralidad del concepto de "interés público" y de la forma gramatical del argumento del "mayor beneficio".

Por último, en cuestiones de fondo, la práctica del órgano de transparencia no le debe mucho a la teoría de los principios, ni al test de proporcionalidad, según la teoría canónica de Robert Alexy. Por esto no deja de ser intrigante que teniendo a su disposición la herramienta argumentativa de la prueba de interés público, el IFAI, y posteriormente el INAI, hubiesen adoptado la herramienta del test de proporcionalidad, y que el legislador haya seguido sin mucha reflexión el mismo camino. ¿Qué podría explicar este curso de acción? En el caso del IFAI-INAI es claro que enfrentaban los ya mencionados problemas de fundamentación para incluir por fuera

52 Ley General de Transparencia y Acceso a la Información Pública, mayo de 2015 , "Artículo 149. El organismo garante, al resolver el recurso de revisión, deberá aplicar una prueba de interés público con base en elementos de idoneidad, necesidad y proporcionalidad, cuando exista una colisión de derechos".

53 R 1655/10 (véase la nota 5), y RDA 4638/12 (véase la nota 6) 
Esta revista forma parte del acervo de la Biblioteca Jurídica Virtual del Instituto de Investigaciones Jurídicas de la UNAM

del marco legal excepciones a la regla general de la confidencialidad de la información personal. Quizá la entronización del test de proporcionalidad para resolver estos problemas, con las fallas que hemos indicado, haya sido el precio que tuvo que pagar el órgano de transparencia para legitimar su conducta. En el caso del legislador podríamos pensar en una inducción al error. Sin muchas posibilidades para abordar debates técnicos, esta decisión parece inducida por los especialistas que elaboraron el proyecto de ley. Es razonable que éstos siguieran el camino abierto por el órgano de transparencia sin suficiente reflexión sobre su utilidad para resolver este tipo específico de problemas.

\section{Conclusión}

La creatividad del órgano de transparencia para exceptuar la regla general de la confidencialidad de la información personal por motivos de interés público abrió el debate sobre los métodos para resolver la publicidad de la información personal en poder del Estado. La ponderación de daños, la prueba de interés público y el test de proporcionalidad entraron a ser parte de los ejercicios argumentativos del órgano de transparencia.

Esta situación, anómala en una cultura jurídica y burocrática tan apegada a la legalidad, llevó al órgano de transparencia a ensayar argumentos de fundamentación y de legitimación de sus prácticas. Para esto fueron clave la lectura constitucional, y no sólo legal, del problema de la publicidad de la información personal en poder del Estado y los conceptos "interés público", "colisión de derechos fundamentales" y "ponderación”.

En el uso de estos conceptos puede verse una buena dosis de creatividad local combinada con elementos de la foránea teoría de los principios, defendida principalmente por Robert Alexy. El órgano de transparencia termina por adoptar un método ecléctico, en donde combina una lectura vernácula de la ponderación a partir del argumento del "mayor beneficio" con los elementos del test de proporcionalidad.

El uso de esta metodología para la solución del problema de la publicidad de la información personal en poder del Estado termina por privilegiar la retórica sobre el fondo. En especial por dos razones: porque el test de proporcionalidad (con sus tres subprincipios) no es una herramienta para resolver colisiones entre derechos fundamentales, y porque la polaridad de las medidas de satisfacción de los derechos en colisión, publicidadconfidencialidad, determina que los análisis de idoneidad y de necesidad sean superfluos. 
Esta revista forma parte del acervo de la Biblioteca Jurídica Virtual del Instituto de Investigaciones Jurídicas de la UNAM

La práctica del órgano de transparencia y el prestigio de dicho test terminaron por impactar la regulación de las excepciones a la confidencialidad de la información personal en poder del Estado en Ley General de Transparencia y Acceso a la Información Pública de 2015. Esta situación, sin embargo, no debe ocultar la necesidad de evaluar la utilidad de esta metodología para resolver el problema de la publicidad de la información personal en poder del Estado, ni tampoco agotar la posibilidad del "equilibrio reflexivo" que, como diría Rorty, parece ser hasta ahora el mejor método para decidir cómo deben gobernarse las sociedades.

\section{Fuentes de información}

Aleinikoff, T. Alexander, 1987, "Constitutional Law in the Age of Balancing”, The Yale Law Journal, vol. 96, núm. 5.

Alexy, Robert, 1997, Teoría de los derechos fundamentales, trad. de Ernesto Garzón Valdés, Madrid, Centro de Estudios Constitucionales.

Alexy, Robert, 2003, "On balancing and Subsumption. A structural comparison”, Ratio Iuris, vol. 16, núm. 4, Dec.

Bernal Pulido, Carlos, 2003, El principio de proporcionalidad y los derechos fundamentales, Madrid, Centro de Estudios Constitucionales.

Carbonell, Miguel (ed.), 2005, El principio de proporcionalidad y la interpretación de la Constitución, Quito, Ministerio de Justicia y Derechos Humanos.

Carbonell, Miguel (ed.), 2008, Neoconstitucionalismo(s), Madrid, Trotta.

JaCkson, Vicki C., 2015, "Constitutional Law in an Age of Proportionality", The Yale Law Journal, vol. 124, núm. 8, junio.

Jansen, Nils, 2011 , "Los fundamentos normativos de la ponderación racional en el derecho", trad. de Daniel Oliver-Lalana, en Jan-R-Sieckmann (edit.), La teoría principialista de los derechos fundamentales, Madrid, Marcial Pons.

Kumm, Mattias, 2011, "Más allá del principio de proporcionalidad. El pluralismo estructural de los derechos fundamentales en el derecho constitucional de los Estados Unidos", trad. de Daniel Oliver-Lalana, en JaN-RSieckmann (edit.), La teoría principialista de los derechos fundamentales, Madrid, Marcial Pons.

López-Ayllón, Sergio y Posadas, Alejandro, 2007, "Las pruebas de daño e interés público en materia de acceso a la información. Una perspectiva comparada”, Derecho Comparado de la Información, núm. 9. 
Esta revista forma parte del acervo de la Biblioteca Jurídica Virtual del Instituto de Investigaciones Jurídicas de la UNAM

Luna Pla, Issa y Ríos Granados, Gabriela, 2010, "El secreto fiscal y el IFAI: primer precedente de apertura por causa de interés público en México", Derecho Comparado de la Información, núm. 16, julio-diciembre.

Mcdonagh, Maeve y Paterson, Moira, 2017, "FOI in the Balance: The Function and Role of the Public Interest Test", Public Law: The Constitutional \& Administrative Law of the Commonwealth, Issue 1, January.

Mendoza Escalante, Mijail, 2007, Conflictos entre derechos fundamentales. Expresión, información y honor, Lima, Palestra.

Nava Tovar, Alejandro, 2015, La institucionalización de la razón. La filosofía del derecho de Robert Alexy, México, Universidad Autónoma Metropolitana.

Schlink, Bernard, 2014, "El principio de proporcionalidad", trad. de Nuria Pastor Muñoz, en Montealegre, Eduardo (comp.), La ponderación en el derecho. Evolución de una teoría, aspectos críticos y ámbitos de aplicación en el derecho alemán, Bogotá, Universidad Externado de Colombia.

Resoluciones del IFAI-INAI

IFAI, R 1326/04 CP María Marván Laborde.

IFAI, R 934/05 CP Juan Pablo Guerrero Amparán.

IFAI, R 412/06 y R 413/06 acumulados CP Alonso Lujambio Irazábal.

IFAI, R 522/06 CP Alonso Lujambio Irazábal.

IFAI, R 425/09 CP Jacqueline Peschard Mariscal.

IFAI, R 3751/09 CP María Marván Laborde.

IFAI, R 6030/09 CP Ángel Trinidad Zaldívar.

IFAI, R 5084/10 CP Jacqueline Peschard Mariscal.

IFAI, R 1655/10 CP Ángel Trinidad Zaldívar.

IFAI, R 751/11 CP Sigrid Arzt Colunga.

IFAI, RDA 0621/12 CP Sigrid Arzt Colunga.

IFAI, RDA 4638/12 CP Ángel Trinidad Zaldívar.

IFAI, RDA 178/13 CP María Elena Perez-Jaén Zermeño.

INAI, RDA $1118 / 15$ CP Rosendoevgueni Monterrey Chepov.

INAI, RDA 1312/15 CP Oscar Mauricio Guerra Ford

INAI, RDA 2165/15 CP Areli Cano Guadiana.

INAI, RDA 2445/15 CP Areli Cano Guadiana.

INAI, RDA 5112/15 CP Areli Cano Guadiana. 
Esta revista forma parte del acervo de la Biblioteca Jurídica Virtual del Instituto de Investigaciones Jurídicas de la UNAM http://www.juridicas.unam.mx/ https://biblio.juridicas.unam.mx/bjv

INAI, RDA 5599/15 CP Ximena Puente de la Mora.

INAI, RDA 6206/15 CP María Patricia Kurzcyn Villalobos.

INAI, RDA 6302/15 CP Areli Cano Guadiana.

INAI, RDA 6531/15 CP Joel Salas Suárez.

INAI, RDA 67/16 CP María Patricia Kurczyn Villalobos. 